\section{Availability of information on reptile health and welfare from stores selling reptiles}

\section{David Williams', Rebecca Jackson ${ }^{1,2}$}

1 Department of Veterinry Medicine, University of Cambridge, Cambridge, UK

2 Vets4Pets veterinary surgery, Inside Pets at Home, Princes Gate Retail Park, Catterick Garrison, UK

Keeping reptiles is becoming more and more popular. Optimising reptile health and welfare depends on understanding of their environmental and nutritional needs. But where does a novice reptile owner obtain such information? This study aimed to investigate what information is available in pet shops and how well this information is imparted.

Fifty pet shops in different locations around the UK were visited. Information on reptile care and welfare was gathered by asking shop assistants a structured set of questions including; which species are recommended for a novice reptile owner? What is the recommended enclosure size and furnishings? What is required regarding thermoregulation, humidity, ultraviolet illumination and nutrition? What is the life expectancy, signs of illnesses and where can one find best available veterinary treatment?

33 of shops were general pet stores, 17 were specific reptile/exotic pet centres. $12 \%$ of shops visited provided a staff-member to answer questions regarding reptiles. The majority recommended bearded dragons, leopard geckos and corn snakes. 52\% suggested bark or woodchips as substrate, 23\% recommended sand, 5\% recommended "reptile carpet', 9\% suggested kitchen roll or newspaper, while $4 \%$ recommended soil. $7 \%$ did not mention substrate. $70 \%$ recommended providing a hiding place for the reptile. All shops recommended providing a heat source. 13\% recommended using two sources but only $8 \%$ suggested providing a temperature gradient. $22 \%$ recommended a device monitoring vivarium temperature. $26 \%$ of shops gave verbal recommendations on humidity while $12 \%$ provided an information sheet detailing humidity requirements. $62 \%$ provided no information. No sellers made any recommendation regarding UV radiation for snakes. $80 \%$ of stores offering bearded dragons recommended UV light use. Only 35\% of those selling leopard geckos advised purchase of a UV source. All sellers appeared to understand the importance of nutrition and the requirements for the species sold but only $12 \%$ recommended a calcium supplement. $88 \%$ of shops gave some information on signs in an unhealthy reptile. Only $12 \%$ were able to recommend a veterinary practice with particular expertise regarding reptile care.

We found excellent information on reptile husbandry in some shops while others offered less advice. It is hoped that through this study stores can recognise best practice so that all shops can aspire to the highest levels of information. We suggest that colaboration between reptile stores and veterinary practices with reptile expertise could be particularly valuable.

\section{Pleural effusion in two rats (Rattus norvegicus) as a complication of dilated cardiomyopathy \\ Sara Dias ${ }^{1}$, Carlo Anselmi², Jaume Martorell ${ }^{3}$}

1 ECZM Resident, Fundaciò Hospital Clìnic Veterinari, Universitat Autònoma de Barcelona, Barcelona, Spain

2 ECVDI Resident, Fundaciò Hospital Clinic Veterinari, Universitat Autònoma de Barcelona, Barcelona, Spain

3 Dipl. ECZM (Small Mammals), Universitat Autònoma de Barcelona, Departament de Medicina i Cirurgia Animals, Barcelona, Spain

\section{INTRODUCTION}

Dilated cardiomyopathy (DCM) is a myocardial disease characterized by primary systolic dysfunction of the left ventricule with secondary eccentric hypertrophy and left atrial dilation. The right heart chambers are variably involved in this disease. Although DCM begins with an asymptomatic phase, of variable duration, it is a progressive and usually fatal disease that leads to congestive heart failure (CHF), arrhytmias, and often sudden cardiac death. Rats are widely used as experimental models but naturally occuring cardiac disease is poorly described.

\section{CLINICAL CASE}

Two 2-year-old female rats (Rattus norvegicus) were presented to the emergency department with evident dyspnea and tachypnea. Chest X-ray showed a bilateral effusion in both animals.

In rat 1 , drainage of the effusion yielded a cloudy, offwhite fluid that settled in layers in the drainage container (16 $\mathrm{ml}$ ). Pleural fluid examination revealed a lymphocyte-rich transudate with high levels of cholesterol (Chol) and triglycerides (Trig) (Chol $27 \mathrm{mg} / \mathrm{dL}$ and Trig $>375 \mathrm{mg} / \mathrm{dL}$ in pleural fluid; Chol $80 \mathrm{mg} / \mathrm{dL}$ and Trig $130 \mathrm{mg} / \mathrm{dL}$ in blood). Ultrasonography (US) and computed tomography (CT) of the thorax and abdomen revealed bilateral pleural effusion, severe dilated cardiomyopathy and mild edematous abdominal effusion. Due to ongoing chylous leak the patient was commenced on a low fatty acid diet, octreotide and heart medication (furosemide, benazepril, pimobendan and digoxin). However, because of worsened of clinical signs the animal was euthanized 5 days later.

In rat 2, drainage of the effusion yielded a translucent liquid with small amount of blood $(3 \mathrm{ml})$. Pleural fluid examination revealed a transudate rich in macrophages and cells compatible with mastocites. Cardiac ultrasonography and CT revealed a severe dilated cardiomyopathy of four chambers without effective contractility and a clot at the level of the aorta. The patient started with cardiac medication and was monitored weekly. Unfortunately the rat died 27 days later.

\section{CONCLUSION}

Many causes have been involved in the development of a DCM, such as chemotherapy, inflammatory process, nutrient deficiencies, but in most cases are considered idiopathic. 